INPLASY

PROTOCOL

To cite: Dong et al. Tuina of post-stroke mental disorders and sleep disorders: a protocol of systematic review and meta-analysis. Inplasy protocol 202070038. doi:

10.37766/inplasy2020.7.0038

Received: 11 July 2020

Published: 11 July 2020

Corresponding author:

Xing Dong

dxtcm20@yeah.net

Author Affiliation:

Chengdu University of TCM

Support: Sichuan Science and technology.

Review Stage at time of this submission: The review has not yet started.

Conflicts of interest:

We declare that we do not have any commercial or associative interest that represents a conflict of interest in connection with the work submitted.

\section{Tuina of post-stroke mental disorders and sleep disorders: a protocol of systematic review and meta-analysis}

Dong, X13 Zhang, R²; Guo, Y3; Chen, LF4; Liu, Y5.

Review question / Objective: Compared with drug therapy alone, the effect of Tuina combined with drug intervention for post-stroke mental disorders and sleep disorders has any changes? Evaluate the safety of Tuina in treating post-stroke mental disorders and sleep disorders.

Condition being studied: Members of the research group have conducted investigations and studies on the integration of "treatment and health maintenance" and old-age undertakings and industrial development in chengdu. Moreover, clinical trials related to intervention of formula in stroke sequelae were carried out. So, we have a certain preliminary basis.

INPLASY registration number: This protocol was registered with the International Platform of Registered Systematic Review and Meta-Analysis Protocols (INPLASY) on 11 July 2020 and was last updated on 11 July 2020 (registration number INPLASY202070038).

\section{INTRODUCTION}

Review question / Objective: Compared with drug therapy alone, the effect of Tuina combined with drug intervention for post- stroke mental disorders and sleep disorders has any changes? Evaluate the safety of Tuina in treating post-stroke mental disorders and sleep disorders. 
Rationale: Stroke is the second greatest cause of death and one of the leading causes of disability. Tuina is a common therapeutic method for ischemic stroke sequelae in China, it has a long history as an effective natural therapy without side effects. But at the present, there is no systematic evaluation report on its therapeutic effectiveness and safety for the treatment of ischemic stroke sequelae.

Condition being studied: Members of the research group have conducted investigations and studies on the integration of "treatment and health maintenance" and old-age undertakings and industrial development in chengdu. Moreover, clinical trials related to intervention of formula in stroke sequelae were carried out. So, we have a certain preliminary basis.

\section{METHODS}

Search strategy: Search dates: from Start of database establishment to 2020.06; Literature sources: The Cochrane Library, MEDLINE (via PubMed), EMbase (via OVID), Web of Science, CNIK, CBM, VIP, etc; Rerun prior to the final analysis.

Participant or population: Included criteria: (1) Patients meeting the diagnosis of stroke sequelae; (2) Adults aged $>18$ years; (3) No national, ethnic or racial restrictions. Exclusion criteria: (1) The subjects were patients in stroke progressing.

Intervention: Tuina was the main intervention measure in the intervention group. Tuina types are not limited, including massage, manipulation, Chinese medicine massage, etc.

Comparator: Tuina was the main intervention measure in the experimental group. The control group could be other drugs, routine treatment, health education or no intervention. Except for the main intervention measures, other intervention measures should be consistent between the experimental group and control group, Tuina type, genre, treatment time unlimited.
Study designs to be included: A randomized controlled trial (RCT), with or without the word "random", should be included in the text. No language and publication time limit.

Eligibility criteria: Randomized controlles trials (RCTs) with no limitations on binding published in English and Chinese up to June 30, 2020 will be included. Patients with primary diseases related PSMDs symptoms will be include, regardless of the underlying diseases, age, gender, education, ethnicity, and occupation. There will be no restrictions regarding language or publication status. Non-randomized trials, case reports, observational studies, and reviews will be excluded.

Information sources: Electronic databases: PubMed, the cochrane Library, web of science, ENASE, ICTRP, Embase, the China National Knowledge Infrastructure (CNKI), and Chinese Biomedical Literature Database (CBM), Wan-fang Data (WANGFANG), Chinese Science and Technology Periodical Database (VIP). Contact with authors: Xing Dong: dxtcm20@yeah.net; Renyan Zhang: 343758213@qq.com; Ying Guo: 83740404672@qq.com; LongFang Chen: missdmx@163.com; Yuan Liu : liuy60s@sina.com

Main outcome(s): Hamilton depression scales, Hamilton anxiety scale, the mental health part of the MOS item short from health survey (SF-36), Generic Quality of Life Inverntory-74, Center for Epidemiologyic Studies Deparession Scale, Pittsburgh sleep quality index.

Additional outcome(s): Adverse events, allcause death during the entire treatment and follow-up period.

Quality assessment / Risk of bias analysis: Two independent reviewers will separately survey methodological quality with the Cochrane risk of bias tool. The conflicts cannot be settled in the review will search consensus for a thrid author as required. Domains need to be evaluated will 
included: sequence generation; allocation concealment; blinding of participants; blinding of outcome assessments; incomplete outcome data. The bias risk for every item will be classed as "low risk of bias", "high risk of bias" or "unclear risk of bias".

Strategy of data synthesis: We will consider whether to conduct meta-analysis according to clinical research. Clinical research includes the research design of intervention method, measurement method, treatment time, and whether the control group chooses the same or not. When a couple of good multiple homogeneity studies are included, we will conduct a meta-analysis with Review Manager 5.3.5. If $12 \quad 50 \%$ were selected. Otherwise, we will not be able to implement the meta-analysis.

Subgroup analysis: If the above clinical trial causes heterogeneity, we will perform a subgroup analysis based on interventions, different controls, treatment duration, and outcome measurements. We'll tabulate the adverse reactions and then make an evaluation.

Sensibility analysis: We will repeat the system evaluation with different types of study, make multiple tests to test the robustness of the review process decisions, which mainly includes the quality of the method, the sample size and the selection of missing data, and we will observe the fluctuation of the results.

Language: English.

Country(ies) involved: China.

Keywords: mental disorders, sleep disorders, stroke, meta-analysis, systematic review, Tuina.

Contributions of each author:

Author 1 - Xing Dong - The author drafted the manuscript.

Author 2 - Renyan Zhang - The author provided statistical expertise.

Author 3 - Ying Guo - The author contributed to the development of the selection criteria, and the risk of bias assessment strategy.

Author 4 - Longfang Chen - The author contributed to the development of the selection criteria, and the risk of bias assessment strategy.

Author 5 - Yuan Liu - The author read, provided feedback and approved the final manuscript. 